\title{
In Vivo Tracking Techniques for Cellular Regeneration, Replacement, and Redirection
}

\author{
Erik H.J.G. Aarntzen ${ }^{1-3}$, Mangala Srinivas ${ }^{1}$, Piotr Walczak ${ }^{4}$, Miroslaw Janowski ${ }^{4-6}$, Arend Heerschap ${ }^{7}$, \\ I. Jolanda M. de Vries ${ }^{1,2}$, Carl G. Figdor ${ }^{1}$, Jeff W.M. Bulte ${ }^{4}$, and Wim J.G. Oyen ${ }^{3}$ \\ ${ }^{1}$ Department of Tumor Immunology, Radboud University Nijmegen Medical Centre, Nijmegen, The Netherlands; ${ }^{2}$ Department of \\ Medical Oncology, Radboud University Nijmegen Medical Centre, Nijmegen, The Netherlands; ${ }^{3}$ Department of Nuclear Medicine, \\ Radboud University Nijmegen Medical Centre, Nijmegen, The Netherlands; ${ }^{4}$ Russell H. Morgan Department of Radiology and \\ Radiological Science, Division of MR Research, Johns Hopkins University School of Medicine, Baltimore, Maryland; ${ }^{5}$ NeuroRepair \\ Department, MMRC, PAS, Warsaw, Poland; ${ }^{6}$ Department of Neurosurgery, MMRC, PAS, Warsaw, Poland; and ${ }^{7}$ Department of \\ Radiology, Radboud University Nijmegen Medical Centre, Nijmegen, The Netherlands
}

\begin{abstract}
Cellular therapy can be defined as the transplantation of living cells for the treatment of medical conditions. Three main objectives of cellular therapy are regeneration of damaged tissue, replacement of function by secretion of biologically active molecules, and redirection of aberrant processes. Given the complex nature of these approaches, in vivo tracking of the transplanted cells is critical to evaluate their potential benefit and to optimize treatment strategies. Recent advances are reviewed that enable in vivo cell tracking as an important adjunct to implement cellular therapy in clinical practice.
\end{abstract}

Key Words: in vivo imaging; tracking; cellular therapy; transplantation; regenerative medicine

J Nucl Med 2012; 53:1825-1828

DOI: 10.2967/jnumed.112.106146

Cellular therapy is the transplantation of living cells for the treatment of a wide variety of medical conditions. The concept of cellular therapy has existed for centuries, starting with the first documented blood transfusion by Jean-Baptiste Denys in June 1667. Technologic advances from preclinical and early clinical studies promise to revolutionize treatment for today's health care problems, for example, autoimmune diseases, ischemic cardiovascular disease, and neurodegenerative disorders. Three main objectives of cellular therapy can be distinguished, each with individual characteristics that require specific imaging features (Fig. 1). Cellular therapy can be used for regeneration, replacement, and redirection. In regeneration, transplanted cells repair damaged tissue, especially in tissues with limited regenerative potential such as cardiac muscle

Received Aug. 6, 2012; revision accepted Oct. 18, 2012.

For correspondence or reprints contact: Eric H.J.G. Aarntzen, Department of Nuclear Medicine, Radboud University Nijmegen Medical Centre, P.O. Box 9101, 6500 HB Nijmegen, The Netherlands.

E-mail: E.Aarntzen@nucmed.umcn.nl

Published online Nov. 9, 2012.

COPYRIGHT (C 2012 by the Society of Nuclear Medicine and Molecular Imaging, Inc. or nervous tissue. In replacement, transplanted cells act as "active drug" containers, replacing lost cells with a specific function, such as hormone production. In redirection, transplanted cells correct aberrant processes, primarily involving the immune system, such as an insufficient immune response to cancer or an overactive immune response against self-antigens in autoimmune diseases.

A major hurdle blocking widespread clinical acceptance of cellular therapy is the fact that the mechanisms underlying success, or failure, are still poorly understood. Optimization of these complex processes would benefit greatly from the development of dedicated metrics. These metrics can be long-term, such as improvement in tissue function or survival, or short-term, such as direct measurements of the transplanted cell numbers, localization, or functionality. Fast metrics that are noninvasive and allow longitudinal or kinetic data are ideal, since they facilitate early treatment modification.

In vivo imaging is considered to be of pivotal importance in designing optimal treatment strategies. The clinically relevant in vivo imaging modalities comprise scintigraphy, SPECT, PET, and MRI. These techniques have recently been described (1).

The ex vivo handling of transplanted cells allows labeling before transfer to the patient, a procedure that is typically performed using MRI and SPECT. The advantage to this procedure is that the effect of the label on the therapeutic cells can be examined before transplantation. To allow longitudinal imaging, labels with relatively long lifetimes are needed, such as metal-based labels for MRI and ${ }^{111}$ In for SPECT. The lifetime of the label is restricted mainly by leakage from the transplanted cells and radioactive decay. In another approach, transplanted cells are labeled in situ and assessed repetitively, typically using PET, with a high sensitivity and short probe lifetimes. Advances in biotechnology now allow the use of imaging reporter genes, such as metal-binding proteins for MRI (2) or intracellular viral enzymes and cell membrane receptors for 
FIGURE 1. Cellular therapy offers hope for treatment of today's most prevalent diseases, including cancer and cardiovascular diseases. Transplanted therapeutic cells essentially perform 3 main roles: regeneration, replacement, and redirection. Imaging protocols should serve different functions in each of these, that is, emphasizing specific aspects of cellular therapy in order to optimize therapeutic strategies. Important factors for consideration are sensitivity, patient discomfort, exposure to radiation, and minimization of cost.

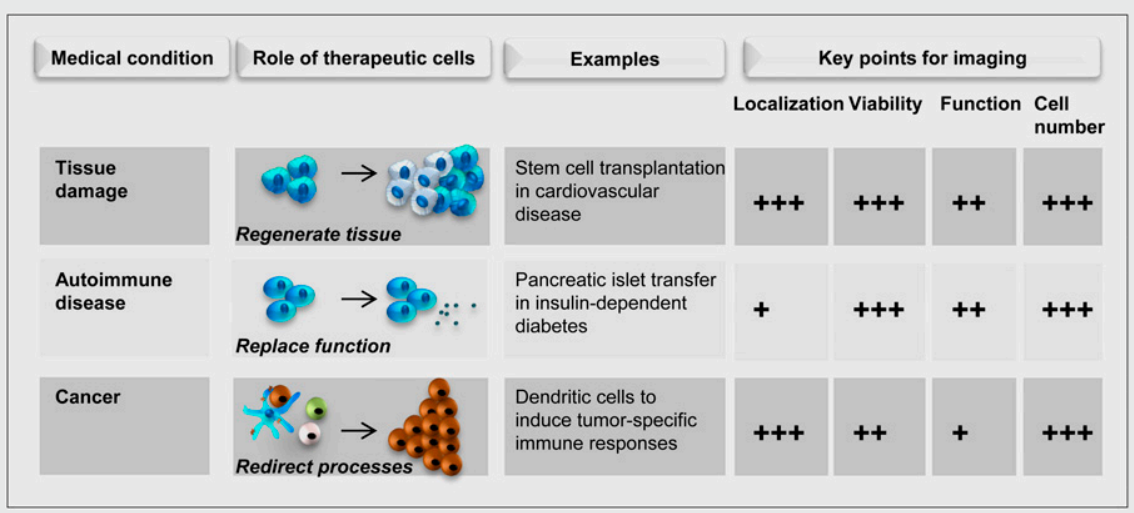

binding radioactive tracers. The main advantage of reporter gene imaging is that only viable cells with intact intracellular machinery can translate the gene into a protein that can be visualized. Furthermore, its transcription can be placed under the control of another gene, allowing transcription only in specific predefined conditions.

In all cases, it is essential to distinguish the relevant cells from all other cells and tissue, that is, specificity and sensitivity must be evaluated (3). Preferentially, an anatomic context is generated to localize the cells in the various tissues. Although this is inherent in MRI, it must be acquired using a complementary modality (typically CT) for SPECT and PET. Restrictions on scanner availability, cost, radiation exposure limits, and patient discomfort should be considered in developing multimodal imaging strategies.

Here, we review upcoming developments that may facilitate translation of cellular therapy to clinical practice.

\section{REGENERATION}

Transplanted stem cells need to relocalize to damaged sites and engraft, proliferate, or differentiate to restore tissue function-an immensely complex process. To date, the main applications are in neurodegenerative diseases (4) and ischemic cardiovascular disease (5), both of which involve tissues with limited regenerative potential. Particularly in cardiovascular disease (6), in vivo tracking of transplanted cells has revealed that only a small percentage of transplanted cells home to the myocardium after intravenous or intracoronary injection (7), partly explaining the limited clinical results. Direct injection into the myocardium resulted in slightly higher numbers of cells in the myocardium, but this technique demands skilled personnel and intracardiac navigation. Furthermore, successful regeneration of myocardium requires that the transplanted cells remain viable and functionally intact. In this respect, the optimal origin and phenotype of transplanted stem cells and the exact mechanism of action are often not known. Although many strategies have been proposed to optimize stem cell engraftment, suitable imaging tools for in vivo cell tracking are essential for proper evaluation (8). Together, the ideal imaging protocol would allow assessment of cell localization, number, viability, and differentiation while allowing for long-term monitoring.

To assess the number and localization of transplanted stem cells, both MRI and scintigraphy/SPECT have commonly been used. The clinical role of MRI in tracking progenitor cells in cardiac diseases is limited (9), although it obviates the use of radioisotopes in cells with high proliferative potential and a long life span. Because SPECT allows dual-isotope imaging, it is often combined with ${ }^{99 \mathrm{~m}} \mathrm{Tc}-$ tetrofosmin to provide information on myocardial viability, motility, and perfusion-important outcome measurements. However, to record long-term cell fate and function, the development of PET reporter genes for radionuclide detection is needed, as described elsewhere (10). Cardiac imaging at high resolution remains technologically challenging, even with electrocardiography triggering.

\section{REPLACEMENT}

From a mechanistic point of view, human cells can function as a versatile exocrine production unit that integrates seamlessly into its natural environment, acting in response to dynamic signals. In this setting, the transplanted cells should be long-lived and perform their function in a stable manner. An example of cellular therapy as a "continuous, active drug infusion" is the transplantation of cadaveric human pancreatic islet cells to replace autologous insulin-producing pancreatic islets in patients with autoimmune diabetes. Despite extensive research, islet graft viability and functionality remain poor. The mechanisms for graft failure include harsh isolation procedures and an inadequate host microenvironment, both of which directly impair the viability of the transplanted cells. Furthermore, immune responses directed against the allogeneic transplanted cells cause a progressive loss of pancreatic islets. Thus, parameters that need to be assessed are viability and the number of transplanted islets, because islet functionality can vary. Issues in imaging islets have been reviewed recently (11).

Using MRI, the fate of labeled transplanted islet cells has been assessed after intraportal infusion in diabetic patients (12). The number of hypointense spots on MRI did not correspond with the number of transplanted cells, nor was there any association with clinical outcome in terms of 
glucose homeostasis (13). On the other hand, MRI cell tracking has been clinically successfully used to qualitatively assess the homing and engraftment of injected dendritic (14) or mesenchymal (15) stem cells. Although superparamagnetic MRI labels, unlike radiolabels, are not restricted by radioactive decay, this method is less suitable for quantification and direct visualization of function (16). A novel class of ${ }^{19} \mathrm{~F}$-based MRI contrast agents with no physiologic background signal has recently been developed (17) and is suitable for quantification directly from the imaging data. Although this technique is under development, the combination of anatomic information and quantitative functional information makes ${ }^{19} \mathrm{~F}$ MRI an attractive modality for in vivo cell tracking.

\section{REDIRECTION}

In contrast to cellular therapies that exploit the exocrine functions of cells, other pathologic processes require direct cell-cell contact and paracrine function, that is, therapeutic cells and target cells need to be near each other. In this case, transplanted cells may be short-lived but perform specialized functions at the relevant site. An example is dendritic cell-based vaccination in cancer patients to induce cancerspecific immune responses, particularly in lymph nodes. It has been previously demonstrated that correct delivery to lymph nodes is crucial for the induction of antitumor responses (Fig. 2) (1). In the case of adoptive T cell cancer therapy, the manipulated cytotoxic $\mathrm{T}$ cells need to home to cancer lesions to exert their function. Improvement of homing of adoptively transferred $\mathrm{T}$ cell lesions translates into an improved clinical outcome (18). Given these considerations, the main parameters that need to be evaluated are the number of cells and the tissue localization of the transplanted cells, with the implication being that labels used for in vivo imaging might be short-lived but quantifiable. The main imaging modalities used in clinical studies are scintigraphy and MRI. The sensitivity of scintigraphic imaging depends on the amount of activity that can be loaded per cell (19) but generally allows quantification of approximately $10^{4}$ labeled cells. The role of scintigraphy in optimizing the route of vaccination in dendritic cell-based vaccination has been described previously (1).

The main drawback of scintigraphy is its lack of anatomic detail. Although lower in cost and widely available, single-modality scintigraphy is increasingly being replaced by SPECT/CT. The radiolabels that are typically used are ${ }^{111} \mathrm{In}$ and ${ }^{99 \mathrm{~m}} \mathrm{Tc}$, with a half-life of $2.8 \mathrm{~d}$ and $6 \mathrm{~h}$, respectively, or ${ }^{18} \mathrm{~F}$, with a half-life of $2 \mathrm{~h}$. These brief half-lives restrict the time in which the radiolabels can be detected in vivo; for dendritic cell-based vaccination this time might be sufficient, whereas in adoptive $\mathrm{T}$ cell transfer this time is much shorter than the lifetime of the transplanted cells. Logistic issues are also introduced, as the entire process from label synthesis to imaging must be performed in a short time. Lastly, label retention within cellular compartments must also be characterized, as imaging modalities typically detect just the label regardless of whether it is contained in the relevant cells, lost to the extracellular matrix, or transferred to other cells. It has become clear that ${ }^{99 \mathrm{~m}} \mathrm{Tc}$ is not as suitable as ${ }^{111} \mathrm{In}$ for labeling cells because of higher leakage of the ${ }^{99 \mathrm{~m}} \mathrm{Tc}$ (20). ${ }^{18}$ F-FDG has proven to be of little value for in vivo tracking because of massive release from the cells (21).

Main challenges in this area of in vivo imaging lie in the combination of exact localization of transplanted cells with simultaneous measurement of their specialized functions. In this respect, PET offers great opportunities; it reveals a 3-dimensional image of functional processes. PET extends the advantages of scintigraphy (quantification) and SPECT/CT (localization) with tracers that can be injected systemically to label the relevant cells in situ, allowing sensitive longitudinal whole-body scans measuring functional processes. For example, melanoma patients have been imaged with $3^{\prime}$-deoxy- $3^{\prime}-{ }^{18}$ F-fluorothymidine PET at various time points to determine the kinetics of dendritic cell-induced immune responses (22). Another interesting approach is exploiting PET to image reporter genes, as recently reviewed by Yaghoubi et al. (10). The first clinical application was in a patient with grade IV glioblastoma multiforme treated with ex vivo expanded autologous cytotoxic T cells, genetically engineered to express

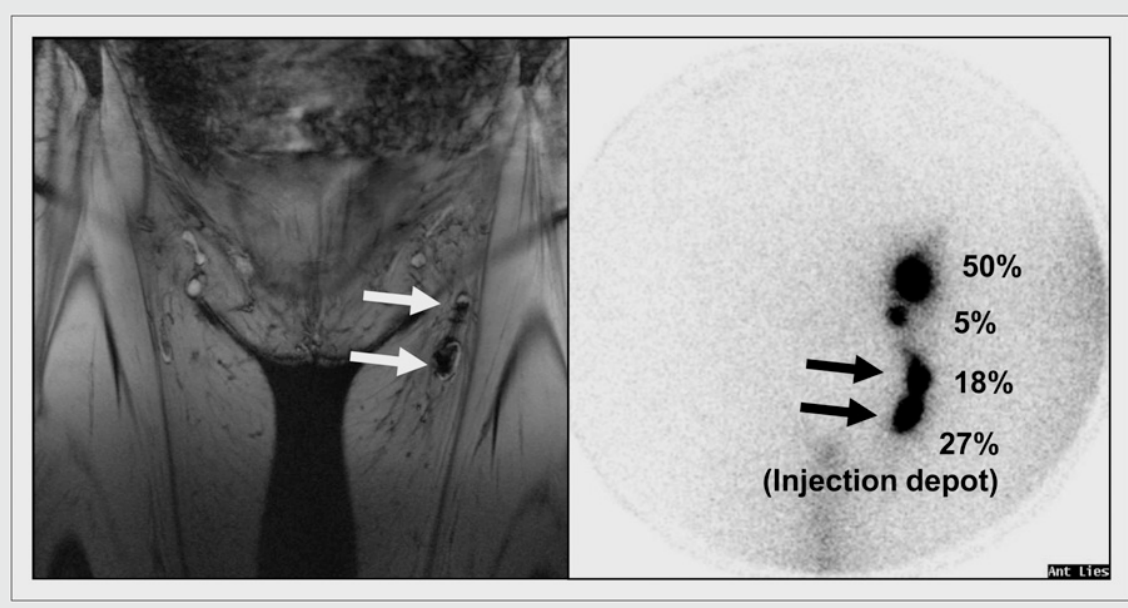

FIGURE 2. In vivo scintigraphy and MRI cell tracking of dendritic cells labeled with superparamagnetic iron oxide and ${ }^{111}$ In after intranodal injection. (Left) Gradient echo coronal MR image showing 2 left inguinal lymph nodes with hypointense signal area $48 \mathrm{~h}$ after injection. (Right) Corresponding scintigraphic image including quantification of relative cellular distribution among multiple lymph nodes. Arrows indicate 2 parts that belong to same lymph node. 
a tumor cell-targeting moiety and the herpes simplex virus 1 thymidine kinase. PET showed that transplanted cells can be traced with 9-(4- ${ }^{18}$ F-fluoro-3-[hydroxymethyl]butyl)guanine. Although safety concerns exist about the viral origin of the reporter genes, techniques are now being developed to circumvent these issues (23). Unfortunately, because of the lack of anatomic information, PET cannot be used for real-time, image-guided injections. With MRI having been successfully used for monitoring accurate cell delivery in real time (24), combined PET/MRI may soon become the preferred in vivo cell-tracking mode.

\section{CONCLUSION}

Because no single imaging modality offers all celltracking options concurrently, multimodal imaging is needed. Imaging strategies to follow cell fate remain to be developed and customized for each individual type of study. This process involves everything from selecting the imaging modality to developing suitable labels and labeling techniques, characterizing labeled cells, imaging, quantifying, and assessing the excretion or metabolic fate of the label. Such studies may be logistically and financially challenging, as they often involve several clinical departments and tight time schedules. However, given the current shift toward tailoring treatments to individual patients and the significant costs involved in cellular therapy, we envision that in vivo cell tracking will become an integral part of clinical cellular therapy.

\section{ACKNOWLEDGMENT}

This work was supported by RO1 NS045062, Netherlands Institute of Regenerative Medicine (NIRM, FES0908), the EU (ENCITE HEALTH-F5-2008-201842), Netherlands Organization for Scientific Research (VENI 700.10.409 and Vidi 917.76.363), ERC (Advanced Grant 269019), and Radboud University Nijmegen Medical Centre (AGIKO2008-2-4). No other potential conflict of interest relevant to this article was reported.

\section{REFERENCES}

1. Srinivas M, Aarntzen EH, Bulte JW, et al. Imaging of cellular therapies. $A d v$ Drug Deliv Rev. 2010;62:1080-1093.

2. Gilad AA, Ziv K, McMahon MT, van Zijl PC, Neeman M, Bulte JW. MRI reporter genes. J Nucl Med. 2008;49:1905-1908.
3. McColgan P, Sharma P, Bentley P. Stem cell tracking in human trials: a metaregression. Stem Cell Rev. 2011;7:1031-1040.

4. Lunn JS, Sakowski SA, Hur J, Feldman EL. Stem cell technology for neurodegenerative diseases. Ann Neurol. 2011;70:353-361.

5. Rasmussen TL, Raveendran G, Zhang J, Garry DJ. Getting to the heart of myocardial stem cells and cell therapy. Circulation. 2011;123:1771-1779.

6. Clifford DM, Fisher SA, Brunskill SJ, et al. Stem cell treatment for acute myocardial infarction. Cochrane Database Syst Rev. 2012;2:CD006536.

7. Abdel-Latif A, Bolli R, Tleyjeh IM, et al. Adult bone marrow-derived cells for cardiac repair: a systematic review and meta-analysis. Arch Intern Med. 2007; 167:989-997.

8. Nguyen PK, Lan F, Wang Y, Wu JC. Imaging: guiding the clinical translation of cardiac stem cell therapy. Circ Res. 2011;109:962-979.

9. Kraitchman DL, Bulte JW. Imaging of stem cells using MRI. Basic Res Cardiol. 2008;103:105-113.

10. Yaghoubi SS, Campbell DO, Radu CG, Czernin J. Positron emission tomography reporter genes and reporter probes: gene and cell therapy applications. Theranostics. 2012;2:374-391.

11. Andralojc K, Srinivas M, Brom M, et al. Obstacles on the way to the clinical visualisation of beta cells: looking for the Aeneas of molecular imaging to navigate between Scylla and Charybdis. Diabetologia. 2012;55:1247-1257.

12. Saudek F, Jirak D, Girman P, et al. Magnetic resonance imaging of pancreatic islets transplanted into the liver in humans. Transplantation. 2010;90:1602-1606.

13. Toso C, Vallee JP, Morel $\mathrm{P}$, et al. Clinical magnetic resonance imaging of pancreatic islet grafts after iron nanoparticle labeling. Am J Transplant. 2008;8:701-706.

14. de Vries IJ, Lesterhuis WJ, Barentsz JO, et al. Magnetic resonance tracking of dendritic cells in melanoma patients for monitoring of cellular therapy. Nat Biotechnol. 2005;23:1407-1413.

15. Karussis D, Karageorgiou C, Vaknin-Dembinsky A, et al. Safety and immunological effects of mesenchymal stem cell transplantation in patients with multiple sclerosis and amyotrophic lateral sclerosis. Arch Neurol. 2010;67:11871194.

16. Kedziorek DA, Muja N, Walczak P, et al. Gene expression profiling reveals early cellular responses to intracellular magnetic labeling with superparamagnetic iron oxide nanoparticles. Magn Reson Med. 2010;63:1031-1043.

17. Srinivas M, Heerschap A, Ahrens ET, Figdor CG, de Vries IJ. ${ }^{19} \mathrm{~F}$ MRI for quantitative in vivo cell tracking. Trends Biotechnol. 2010;28:363-370.

18. Restifo NP, Dudley ME, Rosenberg SA. Adoptive immunotherapy for cancer: harnessing the T cell response. Nat Rev Immunol. 2012;12:269-281.

19. Verdijk P, Scheenen TW, Lesterhuis WJ, et al. Sensitivity of magnetic resonance imaging of dendritic cells for in vivo tracking of cellular cancer vaccines. Int $J$ Cancer. 2007;120:978-984.

20. Blocklet D, Toungouz M, Kiss R, et al. ${ }^{111} \mathrm{In}$-oxine and ${ }^{99 \mathrm{~m}} \mathrm{Tc}-\mathrm{HMPAO}$ labelling of antigen-loaded dendritic cells: in vivo imaging and influence on motility and actin content. Eur J Nucl Med Mol Imaging. 2003;30:440-447.

21. Stojanov K, de Vries EF, Hoekstra D, van Waarde A, Dierckx RA, Zuhorn IS. $\left[{ }^{18} \mathrm{~F}\right]$ FDG labeling of neural stem cells for in vivo cell tracking with positron emission tomography: inhibition of tracer release by phloretin. Mol Imaging. 2012;11:1-12.

22. Aarntzen EH, Srinivas M, De Wilt JH, et al. Early identification of antigenspecific immune responses in vivo by $\left[{ }^{18} \mathrm{~F}\right]$-labeled 3 '-fluoro-3'-deoxythymidine ([18F]FLT) PET imaging. Proc Natl Acad Sci U S A. 2011;108: 18396-18399.

23. Campbell DO, Yaghoubi SS, Su Y, et al. Structure-guided engineering of human thymidine kinase 2 as a positron emission tomography reporter gene for enhanced phosphorylation of non-natural thymidine analog reporter probe. J Biol Chem. 2012;287:446-454.

24. Bulte JW. In vivo MRI cell tracking: clinical studies. AJR. 2009;193:314-325. 